\title{
Developing a mobile application to improve the quality of life among breast cancer survivors
}

Marcelle Dougan ( $\nabla$ marcelle.dougan@sjsu.edu )

San Jose State University https://orcid.org/0000-0003-0538-8887

Tina Korani

San Jose State University

Arden Castle

San Jose State University

Kim Dang

San Jose State University

\section{Research Article}

Keywords: mobile application, breast cancer survivors, quality of life

Posted Date: November 2nd, 2021

DOl: https://doi.org/10.21203/rs.3.rs-992412/v1

License: (c) (i) This work is licensed under a Creative Commons Attribution 4.0 International License. Read Full License 


\section{Abstract \\ Background}

In recent years, there has been a growing recognition of the value of mobile applications for health. The current study explored key factors that drive use of mobile applications among breast cancer survivors,.

\section{Methods}

We conducted in-depth interviews with a convenience sample of 14 ethnically diverse cancer survivors and individuals in their support systems. Interviewees included six breast cancer survivors, one survivor of a different type of cancer, and four individuals in their support systems (caregivers, clinical professionals, or research professionals). Interview transcripts were analyzed using thematic analysis.

\section{Results}

Themes emerged in two overarching categories: 1) finding meaningful support and 2) ensuring utility of a mobile application. In relation to meaningful support, participants identified three important themes: increasing social support and connection, motivation for self-care, and assistance navigating healthcare. In the area of application utility, participants identified three key themes: keeping a user-friendly interface, ensuring credible information, and using the data collected for the purposes intended.

\section{Conclusion}

It is important to consider factors associated with both meaning and utility when developing a mobile application for breast cancer survivors. Further research is needed with breast cancer survivors of additional ethnic minority groups to confirm or extend important areas for consideration.

\section{Introduction}

Routine care for breast cancer patients has traditionally occurred during in-person contact with the primary care provider; however, the use of mobile applications in healthcare has been steadily increasing in recent years [1]. Mobile health (mHealth) applications have the potential to enhance the quality of life for patients with chronic diseases, through improved access to health information and services and ability to promote positive change in health behaviors to prevent acute and chronic diseases, especially in hard-to-reach populations [2, 3]. mHealth apps allow patients with chronic diseases to access health information, self-monitor symptoms, and improve adherence to treatment [4]. For example, mHealth apps have shown great success in improving outcomes of individuals with diabetes, cardiovascular diseases, and cancer [5]. 
Breast cancer (BC) is the most common female cancer worldwide, with nearly 276,500 cases diagnosed in 2020 among women in the United States [6]. The American Cancer Society defines a survivor as "any person with a history of cancer, from the time of diagnosis through the remainder of their life," and due to early-detection and aging of the population, the number of BC survivors increases every year [7]. mHealth apps enable $\mathrm{BC}$ survivors to monitor and record their health routinely, and have the ability to motivate individuals to achieve healthier lifestyles [2,8-10]. Currently, there are a variety of applications for BC patients undergoing treatment, but limited options for women who have completed treatment [1]. Further, many of these focus on one particular aspect such as obesity [11] or exercise [12], and few have been developed specifically for ethnically diverse populations.

Best practices for $\mathrm{BC}$ survivors incorporate healthy eating, active living, emotional resilience, and healthy weight to achieve optimal health for survivors $[13,14]$. Regular physical activity lowers the risk for BC incidence and has an inverse relationship with $B C$ mortality and recurrence [15-17]. Studies among those diagnosed with $\mathrm{BC}$ have shown that regular physical activity can lower the risk of $\mathrm{BC}$ mortality and recurrence [18]. Although exercise is an important protective factor, there are also other factors, mindfulness, that have the potential to lower the risk of $B C$ recurrence $[19,20]$.

The current selection of BC mHealth apps through Google Play and Apple's App Store primarily focus on a singular risk factor, most commonly physical activity. Few BC mHealth apps have incorporated more than one component to account for additional risk factors [21]. This suggests that $B C$ survivors have limited access to apps that address their needs in a holistic manner.

To address these gaps and inform development of future mHealth apps for BC survivors, we conducted a qualitative study to better understand the attitudes and experiences towards mobile applications. The study was designed to explore key factors that drive the use of mobile applications in BC survivors.

\section{Methods}

In-depth qualitative interviews were conducted with cancer survivors and other stakeholders about their attitudes related to mobile health-related applications. A convenience sample of participants was recruited over a seven-month period, from March 2019 to October 2019, using a variety of tools including word of mouth, online methods such as Facebook and Reddit, and through flyers that were posted in community organizations in the San Francisco Bay Area that provide care and support for BC patients. Two additional participants were interviewed in February 2021. Participants included any adult (aged 18 years and older) who expressed interest in participating in the study, and who either previously had been diagnosed with breast or any other cancer or had a family member who was affected by BC, or who had worked with cancer patients in a professional capacity.

The final sample included 14 participants. Most $(n=9)$ were BC survivors, and one was an ovarian cancer survivor. Participants included two individuals with a first degree relative who was a BC survivor, and three experts who provided primary prevention services and/or tertiary care for BC patients. Participants' 
ages ranged between 35 and 75 years. A majority of the participants were white, with 4 participants selfidentifying as persons of color.

Procedures. The study was initially conceived as a series of focus groups. After our first focus group session, in which only 2 participants attended because of scheduling challenges, we proceeded with individual in-depth interviews (IDI). With the exception of one in-person IDI, all interviews took place via the web-based video conferencing tool, Zoom. Interviews were conducted using a semi-structured interview guide, which included questions about participants' current use of mobile applications and their experiences of mobile application use during cancer treatment or recovery. Interviews were recorded, and the data were transcribed, with the exception of one participant who did not wish to have their voice recorded. For this participant, the interviewer recorded the notes by hand. Interview length ranged from 45 minutes to 90 minutes, with most interviews taking around 60 minutes. The study was approved by the Institutional Review Board of San Jose State University and all participants provided informed consent.

Analysis. Thematic analysis, a method of identifying, analyzing, and reporting patterns within qualitative data was used to analyze the data, based on procedures described by Braun and Clarke [22]. The original transcripts of the interviews were first reviewed, and then initial codes based on important emerging ideas and meanings identified by study participants were generated. We then collated codes into potential themes, continuing to review themes to ensure they were reflective of the data. Following this iterative process, we then identified final sub-themes and overarching themes.

\section{Results}

Two themes emerged from participants' interviews with respect to the importance of mobile applications in the recovery phase of women affected by BC: 1) meaning for the recovery process and 2) maximizing potential utility minimizing concerns in relation to practical aspects of the application. Within each of these themes, three sub-themes were identified, and one to three categories identified within each subtheme. Figure 1 summarizes the themes, subthemes, and categories, described in detail below.

\section{Meaning for the Recovery Process}

Three sub-themes emerged in relation to potential meaning for the recovery process associated with individual or family access to an app for managing health after active treatment: 1) finding social connection and support, 2) enhancing motivation and providing positive reinforcement; and 3) navigating through the treatment/recovery process.

\section{Finding social connection and support}

Users perceived a mobile application as a vehicle for connecting with others on a social level and also for giving and receiving support from others. This theme comprised three sub-themes: connecting with others to obtain support, helping others in similar situations, and obtaining information that could help keep the user safe. 
Connecting with others to obtain support, particularly around issues that participants did not necessarily wish to contact a clinical professional about was deemed important. For example, one participant mentioned that some people did not like to talk to therapists and being able to talk about their disease and experiences with respect to their diagnosis in a less formal environment may be helpful. Another participant noted,

"I know that's [mental health] like very important and I know people should have different outlets. This is where they can go because I know some people don't want to go to like therapists. They don't talk to anybody. So, I feel like knowing how to talk about your condition or disease will help out."

Being able to obtain helpful information from other users was also valued, exemplified by one participant who reflected that it would be helpful to connect - not just with other people, but also with a professional for answers to "quick problems and questions".

Although participants found useful information in talking with others, some participants did caution about the need to maintain perspective and avoid fueling worries when reading about others' experience. This was illustrated by one participant who noted,

“...And then you want to be careful because you don't want to run into somebody else's horror story. You might not be heading down that path, but all of a sudden in your brain, you've convinced yourself you are."

During the interviews, many participants described the possibility of helping others in similar situation as beneficial. For example, one participant was diagnosed with BC 20 years ago, when she was in her late twenties observed,

“...And then if I was out of that stage, I would probably, for me, become more of an advocacy position. I was young when I was diagnosed. I wanted to be there for other young patients to say 'you're not alone' ... to even anonymously say 'I'm a real person, and this was my experience, and you'll be okay. You can have kids, you can breastfeed"'

Participants also emphasized the value of information that could keep them safe during treatment and recovery. As one participant noted, people with cancer are immunocompromised and thus must take special care to avoid large crowds that could put them at risk of infection. A mobile application, she noted, could provide information on safe options for purchasing healthier foods in venues such as farmer's markets. Another potential opportunity is in providing current information on potential drug reactions. One participant discovered that she was allergic to a carrier in a drug she normally takes. A mobile application, she reflected, could provide easily accessible information on potential drug reactions.

\section{Enhancing Motivation and Providing Positive Reinforcement}

Several participants noted that using a mobile application during their recovery process might be helpful in staying on track with healthy behaviors for their recovery, through motivational tools that could be 
embedded in the application, such as receiving positive reinforcement and obtaining feedback.

Participants also discussed the importance of getting regular feedback of how well they are doing, or of their progress. For example, one participant stated that during her treatment, not only was it validating to hear from one of her care providers that she was doing a great job, but it was also important to hear specifically where she was doing well.

Almost all participants identified the value of receiving instant feedback on progress in relation to their stated goals, and the accountability that follows from receiving this feedback. For example, one participant stated, "I like the accountability of it. And I like being able to see [my progress].".

However, notably, one participant also pointed out that engagement may be due to the newness of the experience, noting that "Data actually confirms what I should be doing, [it is] motivating, but the glitter runs out of everything eventually..."

\section{Navigating through treatment, recovery, and recurrence}

The third theme that emerged in terms of the potential meaning of a mobile application was the value in navigating the treatment and recovery process. Some participants described the early part of the treatment process, particularly immediately following diagnosis of cancer as one full of uncertainty. With that uncertainty came the need for practical tools to keep track and make sense of substantial new information. In addition, participants noted that the value of the mobile application could change depending on the stage of the treatment process. In navigating treatment in particular, participants talked about the importance of keeping track of medications they needed to take and of their numerous medical appointments, and the need for having a system to manage this. For example, according to one participant,

"When I was undergoing cancer treatment, I think I had four or five different cancer apps. I had one specifically for tracking medication and telling me what each type of medication did, and any concerns with interactions of medications as well as interactions of medication with like herbs and spices...I ended up transferring all of this to a spreadsheet because it was much easier for me to see it and the whole picture."

Some participants emphasized the value of being able to reach out to others for support during their treatment process, and a few mentioned the importance of helping others as they themselves had been helped. Support provided for other participants included navigating the treatment process, or helping create a supportive environment for end-of-life care. This, for example, might be made of several dimensions such as navigating through health care systems and synthesizing (or evaluating) information. According to one participant,

"I like the idea [of an app for my spouse and I to use together] because [spouse] was a huge support to me; he was helpful in some physical ways-just being present and physically taking care of me. One of 
the things that we work on, though, is understanding the emotions of it. He's the kind of person that's straight-ahead, like "Doctor said to do this, Doctor said you're doing great."

Another point raised by this participant was that the mobile application could be helpful in providing resources to the family member or caregiver:

"We had a conversation the other day where he was finally able to hear. [He said] 'Oh, it's not about the medical part, I understand that this is hard for you.'. And that's all I wanted. Yeah, acknowledgement. So maybe an app could help do that in a non-threatening way".

One final sub-theme involved helping coordinate care for others. This sub-theme was illustrated by one survivor who now finds herself helping another $\mathrm{BC}$ patient who is nearing the end of life. She noted that a mobile application would be helpful in helping support systems work together to help coordinate meals and appointments for a friend or family member. She elaborated on the potential of an app to help with both communication and scheduling: "....along with 6 other women, I'm caring for my neighbor. And... we're a village, right...I think add who you want to, and then you have a schedule of who does what..."

\section{Maximizing Potential Utility and Minimizing Concerns}

Three specific sub-themes emerged regarding ways in which a mobile application may be useful: 1) creating a user-friendly interface, 2) ensuring credible information, and 3) minimizing concerns, including ensuring the privacy of information, and transparency about the use of data.

\section{A user-friendly interface}

Participants discussed the importance of a user-friendly interface and often referenced which apps that they currently use were user-friendly. For example, one participant mentioned a media app that lists her most frequently visited podcasts so that whenever she logs on to the website, it is easy to find relevant information. Another user mentioned the importance of receiving the information using limited text to enable ease of use. Another participant mentioned she found some apps unhelpful, and that she was interested in "very simple" basic information and layout.

Several participants discussed the idea of having some control over certain features. Participants mentioned the need to be able to turn off the features they found unhelpful, or to be able to change the appearance of the app if needed. One participant noted that some of the apps tended to overuse the common pink color for $\mathrm{BC}$, and the negative impact it had on her process of moving past the disease, "The ones that have really bright colors and like saccharine colors...I didn't really want to see... but you know this pink oversaturation...that's just a little bit, a little bit too much of a reminder, I think, for me."

Some participants mentioned that while they already use several mobile applications for different services, a helpful feature would be having all the functions of an application for managing their recovery in one location. This idea was typified by one participant, 
"Because something that I've really focused on, and also how my food and exercise are tied together... because right now, I track this in a paper calendar and then I transfer it to an Excel spreadsheet, so that [integration] would be really helpful.".

Another participant also underscored this point, adding that an opportunity for participants to choose some or all of the features to focus on would be an added benefit, noting: "I think everything in one place. I mean if you had the opportunity to focus on and separate what you're looking for then definitely in one place."

\section{Importance of Credible Information}

Participants provided diverse viewpoints on the importance receiving credible information from a mobile application. Two BC survivors who are professionally skilled in research mentioned that they did not necessarily worry about obtaining credible information from the app, given that they possessed the tools needed to seek out such information. In contrast, several other participants noted that the explosion in recent years in the use of social media around health conditions, it was important to ensure that the information was credible. This concern was exemplified by one participant who commented,

"...a lot of times you come up with other people that have asked the same question and you don't know who's answering that question, so you're not sure how reliable that information is. So, a professional coach would be way up there because you know my doctors are really good to respond. But I sometimes feel like, oh, I don't want to bug them with just some educational thing, or you know, why does this [feel bad] here or whatever when it doesn't seem like a big deal."

\section{Ensuring Privacy}

One recurring sub-themes among most participants was privacy concerns related to data collection and use. Although one participant stated she was less worried about privacy, because she assumed that all applicable regulations would be followed, others expressed different privacy-related concerns. For example, one recounted previous negative experience with privacy breaches that caused her to be more cautious with her shared data. Some participants were also concerned about sharing personal information to the public. According to a participant,

"I did have [a few privacy concerns] ...because I did not want to do Facebook. I didn't want to put my stuff out there. When we went through this cancer process, we put nothing online about it. So yeah, of course - it's a private matter to me.... I think my limits would be personal numbers about myself: weight, maybe test results, that kind of thing. It's not so much worrying about privacy as it is managing my personal mental health".

Another participant underscored the importance of transparency as far as what the data collected was being used noting, 
"I know with health care and I'm in healthcare administration, so I know all about HIPAA, but I have never been terribly concerned about privacy. I only don't like my data being abused. Again, like targeting. But I am happy to give information. And security, I'm just not terribly concerned about it... Obviously like anyone else, I want to be protected. As far as health care...but I would be inclined to give whatever was appropriate or useful."

\section{Discussion}

Findings from our qualitative study suggest that women affected by breast cancer value meaningful support through mobile health applications. In addition, design that maximizes utility and minimizes concerns, such as security, is important. The participants from our study suggested that finding social connection and support, enhancing motivation and providing positive reinforcement, and navigating through the treatment/recovery process were integral aspects to address in a successful mobile health application for breast cancer survivors.

Overall, participants noted that using a mobile application could be helpful in improving their adherence to exercise and a healthy diet by providing motivational tools. These findings are consistent with those of Phillips et al [23], who observed that over $80 \%$ of BC survivors would be interested in a remote technologybased intervention that focused on their physical health while about $90 \%$ thought having this support would be beneficial during the recovery process. In addition, about $81 \%$ of survivors were also interested in being able to receive feedback on their physical activity.

Our findings regarding the value of a mobile application in enhancing motivation were consistent with those of Chung et al [24], that found that BC survivors significantly increased their physical activity when they were able to connect with an online community compared to when they were not. An online community that allowed sharing progress on physical activity offered motivation in addition to other activities to further improve personal health. However, there are conflicting studies on whether or not being able to see the progress of others is favored or not [25].

Limitations of our study include the smaller sample size and the relatively low participation among women of color. In addition, the nature of the individual detailed interviews did not allow for additional synergies that could have been realized in a focus group setting. Despite these limitations, our study has several key strengths. To begin with, it is one of the first to examine the attitudes of breast cancer survivors towards mobile applications, following a research-based approach to developing mobile applications for health. In addition, our study covered a broad age range of participants, allowing us to capture participants across the mobile application user spectrum. This holistic approach helps provide perceived relevance to the application and to BC survivors. Users can have different goals and need support in different ways, and personalized experiences can help increase engagement and promote adherence $[25,26]$.

\section{Conclusion}


Although mobile applications are becoming more common, there is a need for consumer perspectives in designing comprehensive mobile applications for breast cancer survivors. Our research has identified the importance of a simplified, responsive, and user-friendly interface. Additional studies incorporating more ethnic minorities could further understand and incorporate the specific needs of these populations.

\section{Declarations}

\section{Acknowledgments}

We wish to thank the participants of the Breast Cancer Wellness Study for their participation in the focus groups and individual detailed interviews.

\section{Statements and Declarations}

Funding: This study was supported by the Central Research Scholarly and Creative Activity grant at San José State University.

Conflicts of interest/Competing interests: none

Ethics approval: this study was approved by the Institutional Review Board at San Jose State University.

Consent to participate: All participants provided informed consent.

Availability of data and material: interview transcripts are available upon request.

Authors' contributions: Marcelle Dougan: Conceptualization, Methodology, Supervision, Investigation, Project administration, Funding acquisition, Writing - original draft, Writing - review \& editing. Tina Korani: Project administration, Methodology, Investigation, Writing - original draft, Writing - review \& editing. Arden Castle: Project administration, Writing - original draft, Writing - review \& editing. Kim Dang: Project administration, Writing - original draft, Writing - review \& editing.

\section{References}

1. Giunti $G$ et al (2018) A biopsy of Breast Cancer mobile applications: state of the practice review. Int J Med Inform 110:1-9

2. Jongerius $C$ et al (2019) Research-Tested Mobile Apps for Breast Cancer Care: Systematic Review. JMIR Mhealth Uhealth 7(2):e10930

3. World Health Organization. mHealth: Use of appropriate digital technologies for public health: report by the Director-General.. 2018; Available from:

https://apps.who.int/gb/ebwha/pdf_files/WHA71/A71_20-en.pdf

4. Hamine $S$ et al (2015) Impact of mHealth chronic disease management on treatment adherence and patient outcomes: a systematic review. J Med Internet Res 17(2):e52 
5. Lee JA et al (2018) Effective behavioral intervention strategies using mobile health applications for chronic disease management: a systematic review. BMC Med Inform Decis Mak 18(1):12

6. National Cancer Institute. Cancer Stat Facts: Female Breast Cancer. 2018; Available from: https://seer.cancer.gov/statfacts/html/breast.html

7. American Cancer Society. Cancer Treatment \& Survivorship Facts \& Figures 2019-2021. 2019; Available from: https://www.cancer.org/content/dam/cancer-org/research/cancer-facts-andstatistics/cancer-treatment-and-survivorship-facts-and-figures/cancer-treatment-and-survivorshipfacts-and-figures-2019-2021.pdf

8. Lyons EJ et al (2016) Testing the effects of narrative and play on physical activity among breast cancer survivors using mobile apps: study protocol for a randomized controlled trial. BMC Cancer 16:202

9. Rosen KD et al (2018) Quality of life among women diagnosed with breast Cancer: A randomized waitlist controlled trial of commercially available mobile app-delivered mindfulness training.

Psychooncology 27(8):2023-2030

10. Stubbins R et al (2018) A Behavior-Modification, Clinical-Grade Mobile Application to Improve Breast Cancer Survivors' Accountability and Health Outcomes. JCO Clin Cancer Inform 2:1-11

11. McCarroll ML et al (2015) Feasibility of a lifestyle intervention for overweight/obese endometrial and breast cancer survivors using an interactive mobile application. Gynecol Oncol 137(3):508-515

12. Harder $\mathrm{H}$ et al (2017) A user-centred approach to developing bWell, a mobile app for arm and shoulder exercises after breast cancer treatment. J Cancer Surviv 11(6):732-742

13. American Institute for Cancer Research. Cancer survival - treatment tips. 2020; Available from: https://www.aicr.org/cancer-survival/treatment-tips/after-treatment/\#1579148064722-5f08ebae2420

14. Bodai BI, Tuso P (2015) Breast cancer survivorship: a comprehensive review of long-term medical issues and lifestyle recommendations. Perm J 19(2):48-79

15. Lahart IM et al (2015) Physical activity, risk of death and recurrence in breast cancer survivors: A systematic review and meta-analysis of epidemiological studies. Acta Oncol 54(5):635-654

16. Loprinzi PD et al (2012) Physical activity and the risk of breast cancer recurrence: a literature review. Oncol Nurs Forum 39(3):269-274

17. Rock CL et al (2020) American Cancer Society guideline for diet and physical activity for cancer prevention. CA Cancer J Clin 70(4):245-271

18. Spei ME et al (2019) Physical activity in breast cancer survivors: A systematic review and metaanalysis on overall and breast cancer survival. Breast 44:144-152

19. Kenne Sarenmalm E et al (2017) Mindfulness and its efficacy for psychological and biological responses in women with breast cancer. Cancer Med 6(5):1108-1122

20. Modica C, Hoenig K (2018) Mindfulness in Follow-Up Care After Breast Cancer: Can It Prevent Recurrence? Breast Care (Basel) 13(2):102-108 
21. Napoles AM et al (2019) Feasibility of a Mobile Phone App and Telephone Coaching Survivorship Care Planning Program Among Spanish-Speaking Breast Cancer Survivors. JMIR Cancer 5(2):e13543

22. Braun V, Clarke V (2006) Using thematic analysis in psychology. Qualitative Research in Psychology 3(2):77-101

23. Phillips SM et al (2017) Breast cancer survivors' preferences for technology-supported exercise interventions. Support Care Cancer 25(10):3243-3252

24. Chung IY et al (2019) Exercise Promotion and Distress Reduction Using a Mobile App-Based Community in Breast Cancer Survivors. Front Oncol 9:1505

25. Monteiro-Guerra F et al., Breast Cancer Survivors' Perspectives on Motivational and Personalization Strategies in Mobile App-Based Physical Activity Coaching Interventions: Qualitative Study. JMIR Mhealth Uhealth, 2020. 8(9): p e18867

26. Roberts AL et al., Breast, Prostate, and Colorectal Cancer Survivors' Experiences of Using Publicly Available Physical Activity Mobile Apps: Qualitative Study. JMIR Mhealth Uhealth, 2019. 7(1): p e10918

\section{Figures}

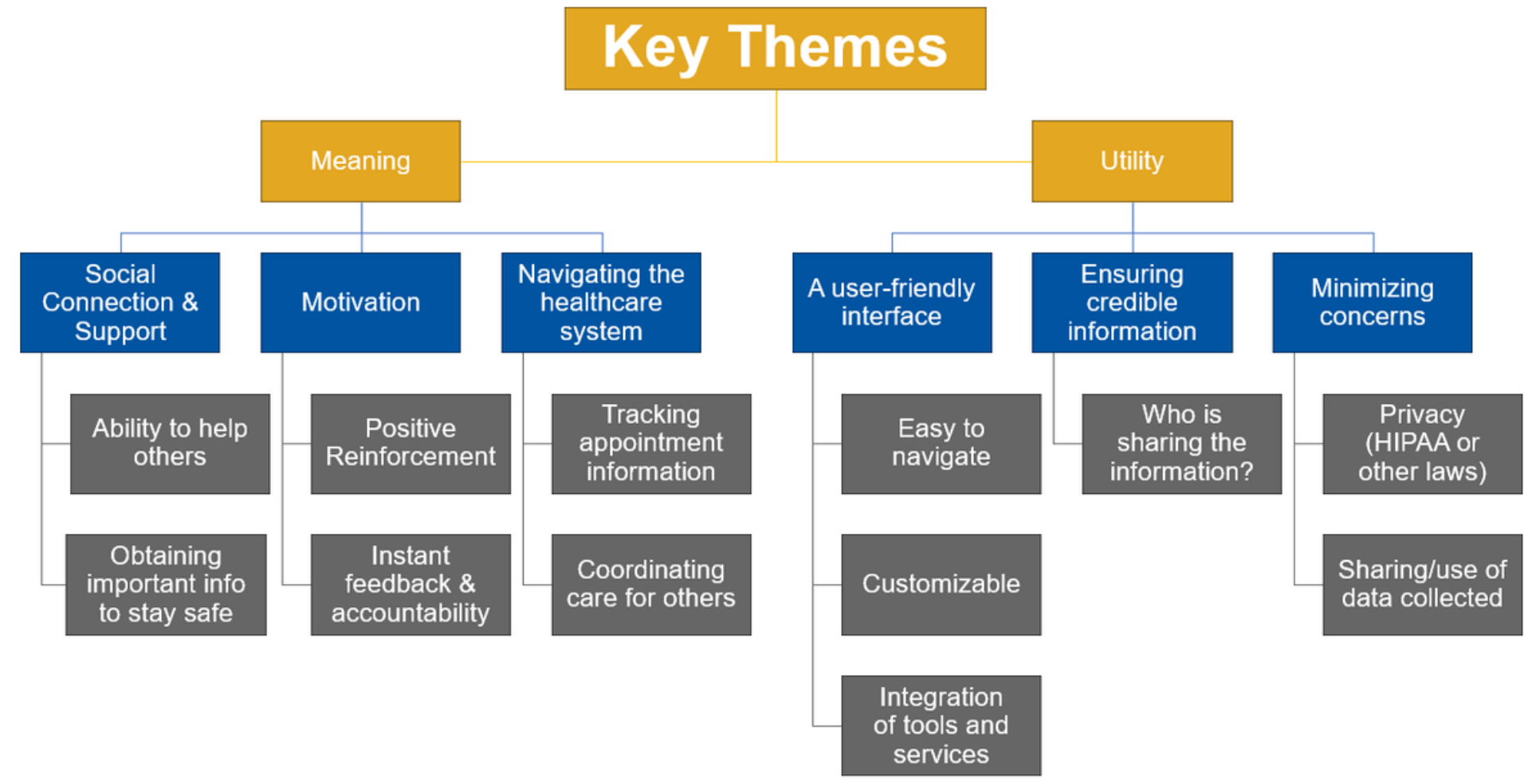

\section{Figure 1}

Identified themes and sub-themes from participant interviews [submitted separately] 